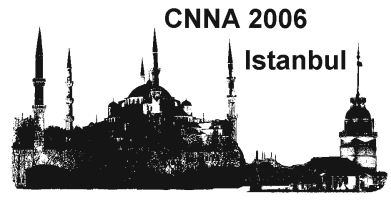

\title{
Testing Pixel Level Snakes
}

\author{
David Lopez Vilariño* and Piotr Dudek ${ }^{\dagger}$ \\ * Department of Electronics and Computer Science, University of Santiago de Compostela, Santiago de Compostela, Spain \\ e-mail: dlv@dec.usc.es

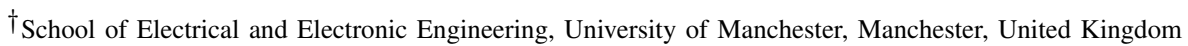 \\ e-mail: p.dudek@manchester.ac.uk
}

\begin{abstract}
Pixel level snakes (PLS) are a cellular active contour technique inspired on the energy-based deformable models. They are based on a pixel-level discretization of the contours and a massively parallel computation on every contour cell which lead to a high speed processing without penalizing the efficiency of the contour location. PLS can be fully implemented in current focal plane processor arrays which make them a real alternative to classical deformable contour approaches.
\end{abstract}

Index Terms-Pixel-Level Snakes, Cellular Active Contours, Active Contours.

\section{INTRODUCTION}

$\mathbf{T}$ He so-called pixel-level snakes (PLS) are a deformable contour technique originally intended to resolve the high computational cost inherent to the classical active contour strategies [1]. They represent a topographic and iterative active contour technique where the contours evolve guided by local information. The inputs consist of a binary image containing the active contours and a multibit image containing information from the image under processing, along with some parameters which configure the control of the contour evolution. The implementation consists of an iterative algorithm which operates along the four cardinal directions performing the contour evolution as a pixel-by-pixel shift (activation and deactivation of pixels of the binary contour image).

PLS have been implemented on hardware architectures with capabilities of SIMD processing, like the CNNUM based chip ACE4K (under the ACE-BOX computing infrastructure [2]) as well as the focal plane processor array SCAMP-3 [3], and they have been tested in some practical applications, including segmentation and tracking of biological structures, moving object segmentation and even for solving the shortest path problem in binary labyrinths [1], [2], [4]. Here we will test PLS in some of these applications. Particularly we will show the segmentation and tracking of different objects (Fig. 1) as well as the estimation of the shortest path between to points in planar binary labyrithns (Fig. 2). To this end we will use both a PC-based simulation and an on-chip implementation (in the SCAMP-3 vision chip) of PLS.

\section{ACKNOWLEDGMENT}

This work was partly supported by Spanish government and Xunta de Galicia through TIC2003-0951 and PGIDIT04PXI20606PN grants.
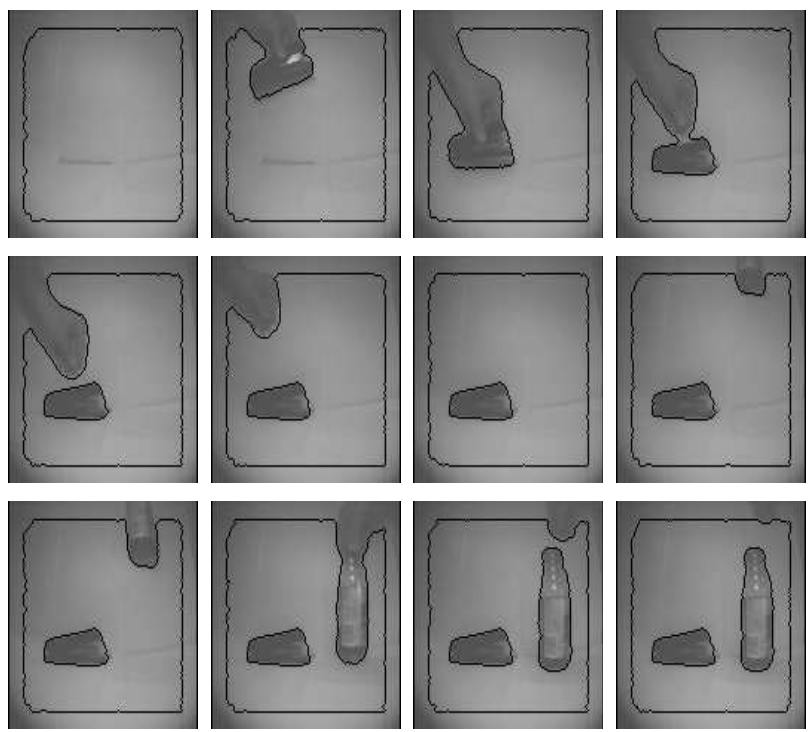

Fig. 1. Several snapshots resulting of the real time processing of PLS on the SCAMP-3 vision chip.
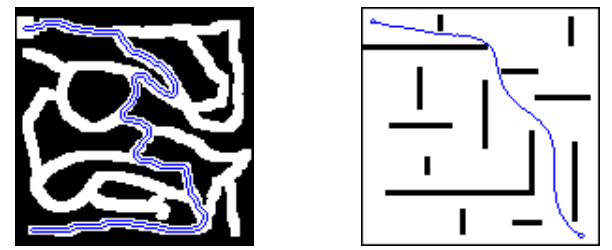

Fig. 2. Examples of shortest path finding with PLS.

\section{REFERENCES}

[1] D. L. Vilariño, D. Cabello, X. M. Pardo and V. M. Brea:"Cellular Neural Networks and Active Contours: A Tool for Image Segmentation, Image and Vision Computing, vol. 21, N. 2, pp. 189-204, 2003.

[2] D.L. Vilariño and Cs. Rekeczky: "Pixel-Level Snakes on the CNNUM: Algorithm Design, On-Chip Implementation and Applications", International Journal of Circuit Theory and Applications, vol. 21, N. 2, pp-189204, 2005.

[3] P.Dudek and S.J. Carey: "General-purpose 128128 SIMD processor array with integrated image sensor", Electronics Letters, Vol. 42, N. 12, p. 678679, 2006.

[4] C. Alonso-Montes, D.L. Vilariño and M.G. Penedo: "CNN-Based Automatic Retinal Vascular Tree Extraction", IEEE Int. Workshop on Cellular Neural Networks and Their Applications, CNNA2005, pp. 61-64 2005. 